5. Burgerhart J. S., et al. Nocturnal and daytime esophageal acid exposure in normal-weight, overweight, and obese patients with reflux symptoms. Eur J Gastroenterol Hepatol. 2014; 26(1):6-10. doi:10.1097/MEG.0b013e328365c3cb.

6. Alves J.R., et al. Diagnosis, treatment and follow-up of barrett's esophagus: a systematic review. Arq Gastroenterol. 2020 Jul-Sep; 57(3):289-295. doi: 10.1590/S0004-2803.

7. Takahashi K., et al. Prevalence of reflux esophagitis in obese Japanese undergoing bariatric surgery. JGH Open. 2019 Dec 17;4(3):519-524. doi: 10.1002/jgh3.12293.

8. Kurin M., Fass R. Management of Gastroesophageal Reflux Disease in the Elderly Patient. Drugs Aging. 2019 Dec; 36(12):1073-1081. doi: 10.1007/s40266-019-00708-2.

9. Zhang L., et al. Survey of nocturnal reflux in patients with gastroesophageal reflux disease in China. J Dig Dis. 2019 Nov; 20(11):589-595. doi: 10.1111/1751-2980.12820.

\title{
Осмонова С.С., Бообекова С.Б., Ташболотова М.Т. \\ Гетерогенные равновесия в тройной системе хлорид европия - никотинамид - вода при 250с и физико-химические свойства твердых фаз
}

Кыргызский национальный университет им. Ж. Баласагына (Кьргызская Республика, Бишкек)

doi $10.18411 / g q-31-03-2021-16$

idsp sciencerussia-31-03-2021-16

\section{Аннотация}

Методом растворимости исследована тройная водная система из хлорида европия и никотинамида при $25^{\circ} \mathrm{C}$. В системе происходит комплексообразование, в результате которого из раствора кристаллизуется новое соединениие состава $\mathrm{EuCl}_{3} \cdot 3 \mathrm{C}_{6} \mathrm{H}_{6} \mathrm{~N}_{2} \mathrm{O} \cdot 6 \mathrm{H}_{2} \mathrm{O}$, конгруэнтно растворимое в воде. Соединение выделено в кристаллическом виде и идентифицировано методом ИК-спектроскопии и дифференциально-термического, рентгенографического анализов.

Ключевые слова: комплексообразование, хлорид европия, никотинамид, конгруэнтно растворимое. ИК-спектроскопия, дифференциально-термический и рентгенографический анализы.

\section{Abstract}

A triple aqueous system of europium chloride and nicotinamide at $25^{\circ} \mathrm{C}$ was investigated. Complexation occurs in the system, as a result of which a new compound of the composition $\mathrm{EuCl}_{3} \cdot 3 \mathrm{C}_{6} \mathrm{H}_{6} \mathrm{~N}_{2} \mathrm{O} \cdot 6 \mathrm{H}_{2} \mathrm{O}$, crystallizes, congruently soluble in water. The compound was isolated in crystalline form and identified by IR spectroscopy and differential thermal, X-ray analyzes.

Key words: complexation, europium chloride, nicotinamide, congruently soluble, IR spectroscopy, differential thermal and X-ray analyzes.

\section{Введение}

Синтез комплексных соединений редкоземельных элементов с органическими лигандами представляет определенный теоретический интерес в связи с изучением их строения и способа координации $[1,2]$.

\section{Экспериментальная часть}

Система хлорид европия - никотинамид - вода изучалась изотермическим методом растворимости. Равновесие устанавливалось в течении 2-3 суток. Химический анализ проводили на ионы европия трилонометрическим методом [3], а азот никотинамида определяли известным методом Къельдаля [4].

\section{Система $\mathrm{EuCl}_{3}-\mathrm{C}_{6} \mathrm{H}_{6} \mathrm{~N}_{2} \mathrm{O}-\mathrm{H}_{2} \mathrm{O}$ при $25^{\circ} \mathrm{C}$}

Экспериментальные данные по изучению изотермы растворимости системы: хлорид европия - никотинамид - вода при $25^{\circ} \mathrm{C}$ приведены в таблице 1 и представлены на диаграмме (рис.1). 


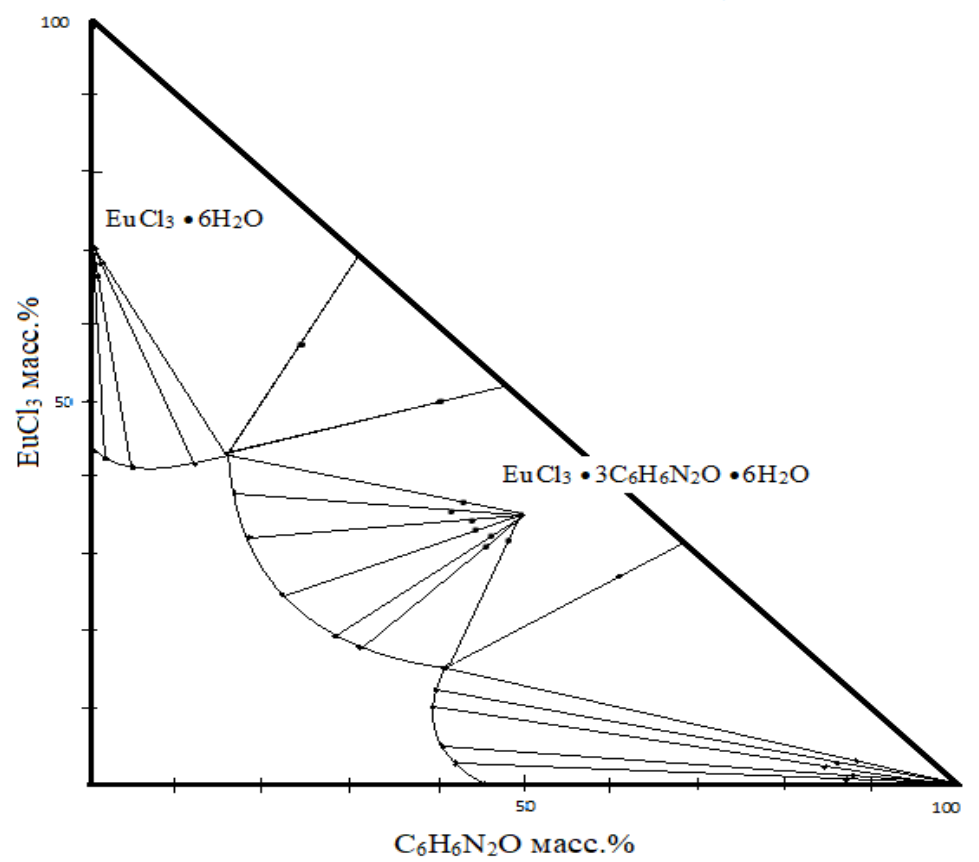

Рис. 1. Изотерма растворимости системы $\mathrm{EuCl}_{3}-\mathrm{C}_{6} \mathrm{H}_{6} \mathrm{~N}_{2} \mathrm{O}-\mathrm{H}_{2} \mathrm{O}$ при $25^{\circ} \mathrm{C}$

Диаграмма растворимости данной системы характеризуется тремя ветвями, разграниченными тремя переходными точками. Из насыщенных растворов, с концентрацией исходных компонентов $43.26 \%$ хлорида европия, $15.96 \%$ амида никотиновой кислоты, кристаллизуется шестиводный хлорид европия, которому отвечает первая ветвь кристаллизации.

Таблий 1

Экспериментальные данные по растворимости в системе $\mathrm{EuCl}_{3}-\mathrm{C}_{6} \mathrm{H}_{6} \mathrm{~N}_{2} \mathrm{O}-\mathrm{H}_{2} \mathrm{O}$ при $25^{\circ} \mathrm{C}$

\begin{tabular}{|c|c|c|c|c|c|}
\hline \multirow[t]{2}{*}{ № } & \multicolumn{2}{|c|}{$\begin{array}{c}\text { Состав жидкой фазы, в } \\
\text { масс. } \%\end{array}$} & \multicolumn{2}{|c|}{$\begin{array}{c}\text { Состав твердой фазы, в } \\
\text { масс. } \%\end{array}$} & \multirow[t]{2}{*}{ Кристаллизующаяся фаза } \\
\hline & $\mathrm{EuCl}_{3}$ & $\mathrm{C}_{6} \mathrm{H}_{6} \mathrm{~N}_{2} \mathrm{O}$ & $\mathrm{EuCl}_{3}$ & $\mathrm{C}_{6} \mathrm{H}_{6} \mathrm{~N}_{2} \mathrm{O}$ & \\
\hline 1 & 43,46 & - & 70,52 & - & \multirow{5}{*}{$\mathrm{EuCl}_{3} \cdot 6 \mathrm{H}_{2} \mathrm{O}$} \\
\hline 2 & 42,65 & 1,89 & 70,48 & 0,15 & \\
\hline 3 & 41,81 & 4,81 & 70,45 & 0,25 & \\
\hline 4 & 41,98 & 12,08 & 70,41 & 0,35 & \\
\hline 5 & 43,26 & 15,96 & 70,39 & 0,45 & \\
\hline 6 & 42,31 & 16,01 & 57,98 & 24,26 & \multirow{2}{*}{$\mathrm{EuCl}_{3} \cdot 6 \mathrm{H}_{2} \mathrm{O}+\mathrm{EuCl}_{3} \cdot 3 \mathrm{C}_{6} \mathrm{H}_{6} \mathrm{~N}_{2} \mathrm{O} \cdot 6 \mathrm{H}_{2} \mathrm{O}$} \\
\hline 7 & 43,19 & 16,04 & 49,81 & 40,02 & \\
\hline 8 & 43,11 & 16,05 & 36,82 & 42,12 & \multirow{4}{*}{$\mathrm{EuCl}_{3} \cdot 3 \mathrm{C}_{6} \mathrm{H}_{6} \mathrm{~N}_{2} \mathrm{O} \cdot 6 \mathrm{H}_{2} \mathrm{O}$} \\
\hline 9 & 38,15 & 16,81 & 44,63 & 41,81 & \\
\hline 10 & 32,75 & 18,05 & 34,36 & 42,93 & \\
\hline 11 & 25,15 & 21,83 & 32,66 & 44,15 & \\
\hline 12 & 19,41 & 28,12 & 33,28 & 45,31 & \\
\hline 13 & 17,98 & 30,88 & 30,45 & 46,17 & \\
\hline 14 & 15,55 & 40,49 & 31,54 & 48,83 & \\
\hline 15 & 15,48 & 40,45 & 27,01 & 61,82 & $\mathrm{EuCl}_{3} \cdot 3 \mathrm{C}_{6} \mathrm{H}_{6} \mathrm{~N}_{2} \mathrm{O} \cdot 6 \mathrm{H}_{2} \mathrm{O}+\mathrm{C}_{6} \mathrm{H}_{6} \mathrm{~N}_{2} \mathrm{O}$ \\
\hline 16 & 15,41 & 40,41 & 3,05 & 88,26 & \multirow{6}{*}{$\mathrm{C}_{6} \mathrm{H}_{6} \mathrm{~N}_{2} \mathrm{O}$} \\
\hline 17 & 12,97 & 39,86 & 2,97 & 86,12 & \\
\hline 18 & 10,06 & 39,48 & 2,28 & 84,97 & \\
\hline 19 & 5,01 & 40,68 & 1,02 & 88,01 & \\
\hline 20 & 2,98 & 42,01 & 0,35 & 87,02 & \\
\hline 21 & - & 45,67 & - & - & \\
\hline
\end{tabular}


При достижении концентрации хлорида европия - 43,11\%, амида никотиновой кислоты - 16,05\%, воды - 40,84\%, из раствора начинает кристаллизоваться новое, конгруэнтно растворимое в воде соединение состава $\mathrm{EuCl}_{3} \cdot 3 \mathrm{C}_{6} \mathrm{H}_{6} \mathrm{~N}_{2} \mathrm{O} \cdot 6 \mathrm{H}_{2} \mathrm{O}$ (вторая ветвь кристаллизации).

Третья ветвь кристаллизации соответствует кристаллизации чистого амида никотиновой кислоты.

Таким образом, при изучении тройной системы $\mathrm{EuCl}_{3}-\mathrm{C}_{6} \mathrm{H}_{6} \mathrm{~N}_{2} \mathrm{O}-\mathrm{H}_{2} \mathrm{O}$ при $25^{0} \mathrm{C}$ мы пришли к выводу, что между реагирующими компонентами происходит химическое взаимодействие, в результате которого образуется новое комплексное соединение с молекулярным соотношением хлорида европия, никотинамида, воды $1: 3: 6$.

Для идентификации соединения были сняты ИК-спектры поглощения (рис. 2, 3), дериватограмма (рис. 4) и рентгенограмма (рис. 5).

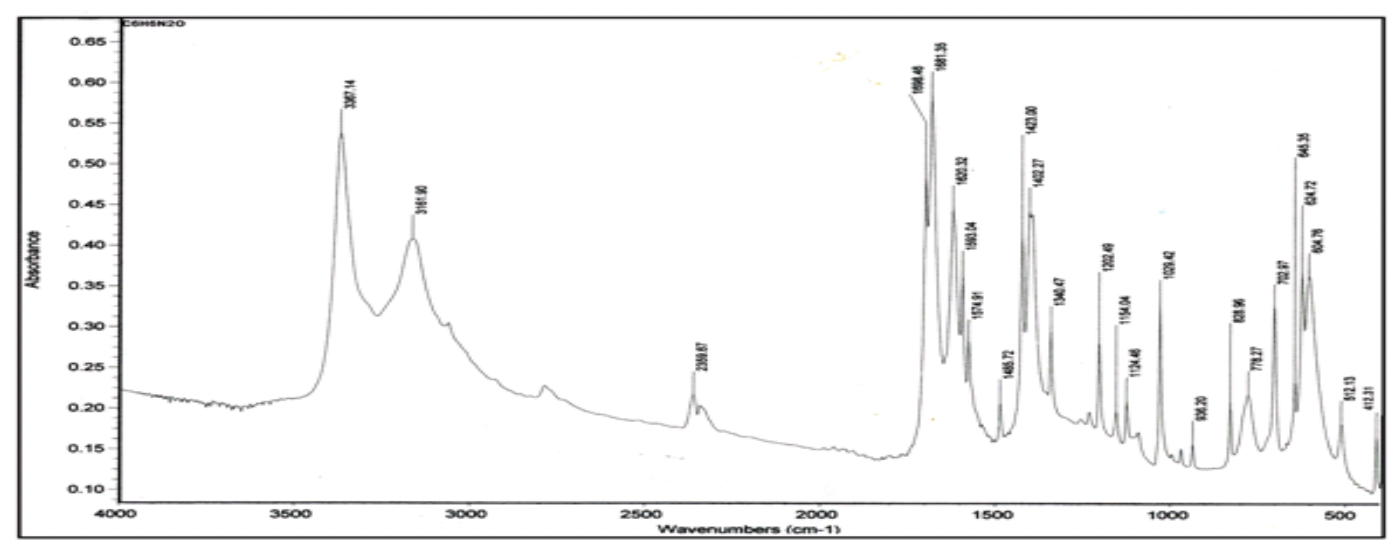

Рис. 2. ИК-спектр поглощееня никотинамида

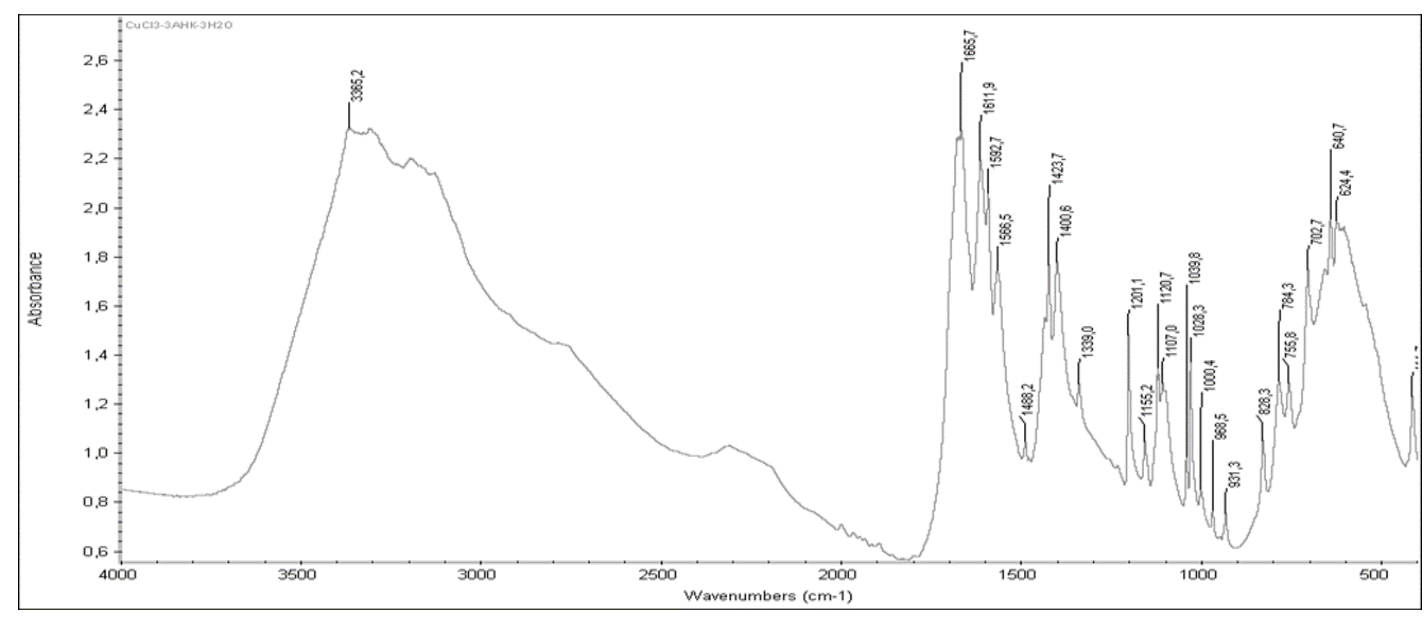

Pис. 3. ИК-спектр поглощуения комплекса $\mathrm{EuCl}_{3} \cdot 3 \mathrm{C}_{6} \mathrm{H}_{6} \mathrm{~N}_{2} \mathrm{O} \cdot 6 \mathrm{H}_{2} \mathrm{O}$

С целью определения места локализации химической связи никотинамида в комплексе исследованы ИК - спектры поглощения никотинамида и его координационного соединения с хлоридом европия в области 400-4000 см-1 на спектрометре “Nicolet-IR-1200» в виде таблеток с бромистым калием. Значения характеристических частот на ИК - спектре поглощения приведены в табл. 2.

В ИК-спектре комплексного соединения $\mathrm{EuCl}_{3} \cdot 3 \mathrm{C}_{6} \mathrm{H}_{6} \mathrm{~N}_{2} \mathrm{O} \cdot 6 \mathrm{H}_{2} \mathrm{O}$ полосы валентного колебания кольца при $1593 \mathrm{~cm}^{-1}$ не претерпевают изменений (изменение частот при этом должно быть в сторону высоких частот), $v$ (кольца) при $1485 \mathrm{~cm}^{-1}$ изменяется незначительно до $1488 \mathrm{~cm}^{-1}$. Но, это не позволяет 
Таблииа 2

Экспериментально полученные частоты валентных и деформащионных колебаний амида никотиновой кислоты и нового полученного соединения

\begin{tabular}{|c|c|c|}
\hline Предполагаемое отнесение & $\begin{array}{c}\text { Полосы поглощения в амиде } \\
\text { никотиновой кислоты, } \mathrm{cm}^{-1} \\
\mathrm{C}_{6} \mathrm{H}_{6} \mathrm{~N}_{2} \mathrm{O}\end{array}$ & $\begin{array}{c}\text { Полосы поглощения в новом } \\
\text { соединении, } \mathrm{cm}^{-1} \\
\mathrm{EuC1}_{3} \cdot 3 \mathrm{C}_{6} \mathrm{H}_{6} \mathrm{~N}_{2} \mathrm{O}^{-1} 6 \mathrm{H}_{2} \mathrm{O}\end{array}$ \\
\hline$v_{\text {as }}\left(\mathrm{NH}_{2}\right), v\left(\mathrm{OH}^{-}\right)$ & 3367,1 & 3365,2 \\
\hline$v_{\mathrm{s}}\left(\mathrm{NH}_{2}\right)$ & 3162 & - \\
\hline$v(\mathrm{C}=\mathrm{O})$ & 1698 & 1666 \\
\hline$\delta\left(\mathrm{NH}_{2}\right), \delta\left(\mathrm{H}_{2} \mathrm{O}\right)$ & 1682 & 1612 \\
\hline$v($ кольца) & 1619 & 1592,7 \\
\hline$v($ кольца), $\delta(\mathrm{CCH})$ & 1593 & 1566,5 \\
\hline$v(\mathrm{CN})$ & 1574 & 1488,2 \\
\hline$\delta(\mathrm{CCH})$ & 1485,7 & 1339 \\
\hline$v($ кольца) & 1340 & 1201 \\
\hline$v(\mathrm{CC}), \delta(\mathrm{CCC})$ & 1202 & 1028,3 \\
\hline$\delta(\mathrm{CCN}), \delta(\mathrm{CO})$ & 1029 & 828,3 \\
\hline$\delta(\mathrm{CO}), \delta(\mathrm{CNC})$ & 829 & 703 \\
\hline & 703 & 640,7 \\
\hline
\end{tabular}

сделать вывод об участии атома азота гетероцикла в координационной связи с металлом [5].

Наблюдается понижение частот валентных колебаний $\mathrm{C}=\mathrm{O}[v(\mathrm{C}=\mathrm{O})]$ от 1681,7 $\mathrm{cm}^{-1}$ до 1665,7 $\mathrm{cm}^{-1}$ по сравнению с некоординированным никотинамидом. Такие изменения позволяют говорить о координации лиганда к иону европия через атом кислорода $\mathrm{C}=\mathrm{O}$ группы.

Таким образом, анализ ИК-спектров никотинамида и нового комплексного соединения показал, что молекулы никотинамида в данном комплексе выступает в роли монодентатного лиганда, координируясь через атом кислорода карбонильной группы амида никотиновой кислоты.

Термографический анализ выполнен на дериватографе Q-1000D. Термическое разложение соединения $\mathrm{EuCl}_{3} \cdot 3 \mathrm{C}_{6} \mathrm{H}_{6} \mathrm{~N}_{2} \mathrm{O} \cdot 6 \mathrm{H}_{2} \mathrm{O}$ протекает в несколько стадий (рис. 4, табл. 3).

При первых двух эндотермических эффектах происходит процесс дегидратации соединения. Убыль массы при этих температурах $\left(90^{\circ} \mathrm{C}\right.$ и $\left.160^{\circ} \mathrm{C}\right)$ составляет соответственно $3 \%$ и $10 \%$ от исходной навески. При повышении температуры до $340^{\circ}$ и $485^{\circ} \mathrm{C}$ наблюдается отщепление молекул никотинамида. Потеря массы при этом составляет $44,25 \%$ от взятой массы и соответствует трем молекулам лиганда. При $340^{\circ} \mathrm{C}$ удаляется две молекулы лиганда, а при $485^{\circ} \mathrm{C}$ одна молекула лиганда, и это сопровождается горением продуктов распада.

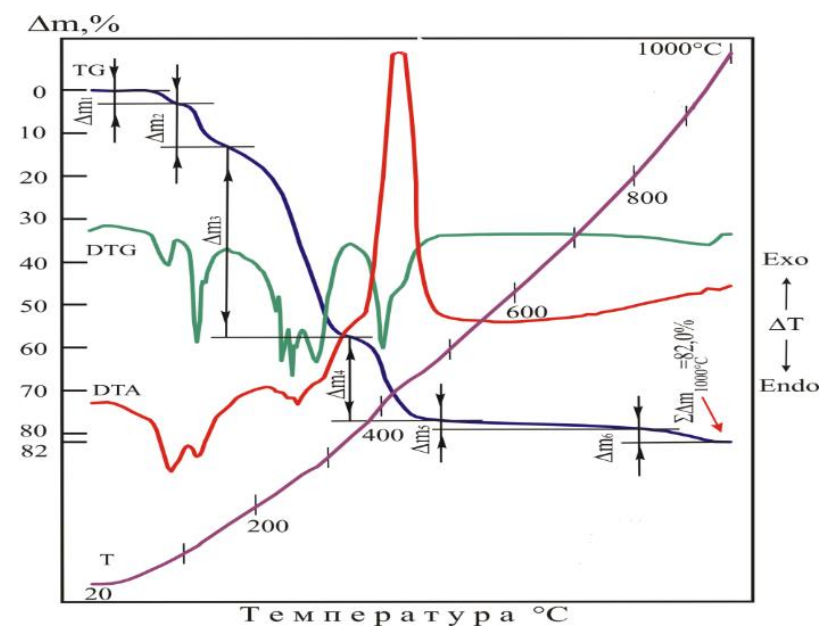

Puc. 4. Дериватограмма соединения $\mathrm{EuCl}_{3} \cdot 3 \mathrm{C}_{6} \mathrm{H}_{6} \mathrm{~N}_{2} \mathrm{O} \cdot 6 \mathrm{H}_{2} \mathrm{O}$ 
Заключающей стадией термического преобразования испытываемого образца является эндотермическая реакция, связанная с окислением неорганической соли, при которой в интервале $485-805^{\circ} \mathrm{C}$ теряется $2,0 \%$ массы исходного образца.

Таблица 3

Данные термического анализа соединения $\mathrm{EuCI}_{3} \cdot 3 \mathrm{C}_{6} \mathrm{H}_{6} \mathrm{~N}_{2} \mathrm{O} \cdot 6 \mathrm{H}_{2} \mathrm{O}$

\begin{tabular}{|c|c|c|c|}
\hline Соединение & $\begin{array}{c}\text { Температура } \\
\text { термоэффекта, } \\
{ }^{\circ} \mathrm{C} \\
\end{array}$ & $\begin{array}{c}\text { Убыль массы, } \\
\%\end{array}$ & $\begin{array}{c}\text { Процессы, происходящие } \\
\text { при нагревании } \\
\text { соединения }\end{array}$ \\
\hline \multirow{6}{*}{$\mathrm{EuCl}_{3} \cdot 3 \mathrm{C}_{6} \mathrm{H}_{6} \mathrm{~N}_{2} \mathrm{O} \cdot 6 \mathrm{H}_{2} \mathrm{O}$} & $20-90$ & 3,0 & \multirow{2}{*}{$\begin{array}{c}\text { Выход механически } \\
\text { связанной воды (процесс } \\
\text { дегидратации) }\end{array}$} \\
\hline & $90-160$ & 10 & \\
\hline & $160-340$ & 44,25 & \multirow{2}{*}{$\begin{array}{c}\text { Разложение органической } \\
\text { части молекулы }\end{array}$} \\
\hline & $340-485$ & 19,5 & \\
\hline & $485-805$ & 2,0 & \multirow{2}{*}{$\begin{array}{c}\text { Окисление и } \\
\text { термодеструкция } \mathrm{EuCI}_{3}\end{array}$} \\
\hline & $805-985$ & 3,25 & \\
\hline
\end{tabular}

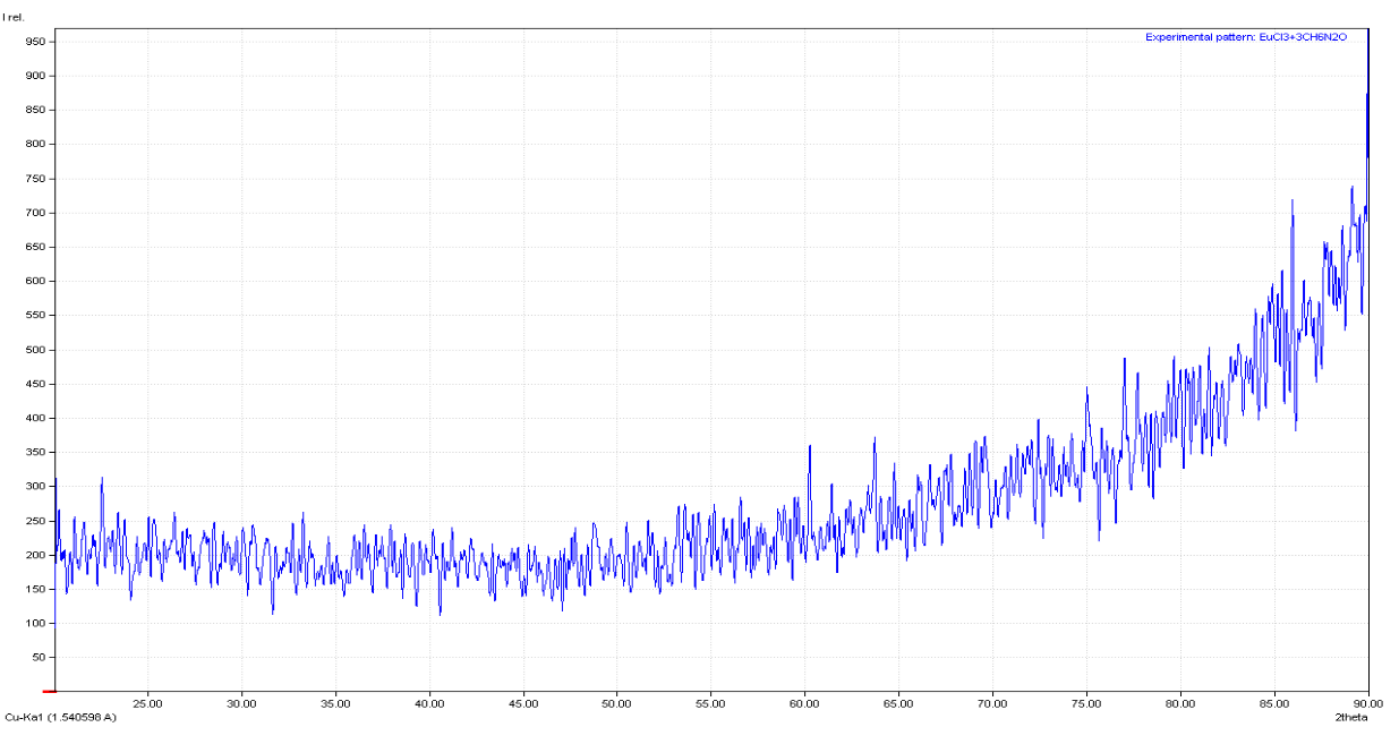

Pис. 5. Схема строения соединения $\mathrm{EuCl}_{3} \cdot 3 \mathrm{C}_{6} \mathrm{H}_{6} \mathrm{~N}_{2} \mathrm{O} \cdot 6 \mathrm{H}_{2} \mathrm{O}$

Процессу деструкции хлорида европия в промежутке $805-985^{\circ} \mathrm{C}$ предшествует потеря веса пробы, равной 3,25\%.

Рентгенографическое исследование комплексного соединения европия с амидом никотиновой кислоты проведено на дифрактометре ДРОН-3.0 (CoК $\alpha$ - излучение, с фильтром). Данные представлены на рис. 5., табл. 4.

Таблий 4

Рентгенографический анализ комплексного соединения $\mathrm{EuCl}_{3} \cdot 3 \mathrm{C}_{6} \mathrm{H}_{6} \mathrm{~N}_{2} \mathrm{O} \cdot 6 \mathrm{H}_{2} \mathrm{O}$

\begin{tabular}{|c|c|c|c|c|c|c|c|c|}
\hline № & $2 \theta$ & $\mathrm{I}(\%)$ & $\theta$ & D (экспер.) & $\mathrm{h}$ & $\mathrm{k}$ & 1 & Сингония \\
\hline 1 & 76,98 & 27 & 38,49 & 1,237672211 & 1 & 0 & 0 & \multirow{13}{*}{$\begin{array}{c}\text { Моноклинная } \\
\mathrm{a}=1,637 \AA \\
\mathrm{b}=1,665 \AA \\
\mathrm{c}=1,6901 \AA \\
\beta=99^{0}\end{array}$} \\
\hline 2 & 85,26 & 27 & 42,63 & 1,137374901 & 0 & 0 & 1 & \\
\hline 3 & 85,46 & 29 & 42,73 & 1,135224203 & 0 & 1 & 0 & \\
\hline 4 & 85,62 & 26 & 42,81 & 1,133511974 & 0 & 0 & 2 & \\
\hline 5 & 85,94 & 27 & 42,97 & 1,130109558 & 0 & 1 & 1 & \\
\hline 6 & 85,98 & 29 & 42,99 & 1,129686310 & 0 & 2 & 0 & \\
\hline 7 & 86,76 & 32 & 43,38 & 1,121523064 & 1 & 2 & 0 & \\
\hline 8 & 87,62 & 28 & 43,81 & 1,112717528 & 2 & 0 & 1 & \\
\hline 9 & 87,84 & 31 & 43,92 & 1,110497122 & 2 & 1 & 1 & \\
\hline 10 & 87,98 & 30 & 43,99 & 1,109090870 & 0 & 1 & 2 & \\
\hline 11 & 88,08 & 28 & 44,04 & 1,108089594 & 1 & 1 & 1 & \\
\hline 12 & 88,26 & 26 & 44,13 & 1,106293966 & 0 & 0 & 3 & \\
\hline 13 & 88,66 & 26 & 44,33 & 1,102334152 & 0 & 3 & 1 & \\
\hline
\end{tabular}




\begin{tabular}{|l|c|l|l|l|l|l|l|l|}
\hline 14 & 88,82 & 27 & 44,41 & 1,100761900 & 1 & 3 & 0 & \\
\cline { 1 - 7 } 15 & 88,98 & 31 & 44,49 & 1,099196266 & 3 & 0 & 0 \\
\hline 16 & 89,14 & 32 & 44,57 & 1,097637216 & 1 & 1 & 0 \\
\hline 17 & 89,36 & 28 & 44,68 & 1,095504211 & 2 & 1 & 0 \\
\hline 18 & 89,56 & 26 & 44,78 & 1,093575788 & 1 & 1 & 2 & \\
\cline { 1 - 6 } 19 & 90 & 31 & 45,00 & 1,089368707 & 1 & 1 & 0 & \\
\hline
\end{tabular}

Таким образом, в рентгенограмме комплекса имеются характерные линии, которые хорошо индицируются в моноклинной сингонии.

Исходя из вышеизложенного, предполагаемое строение комплексного соединения можно представить следующим образом:

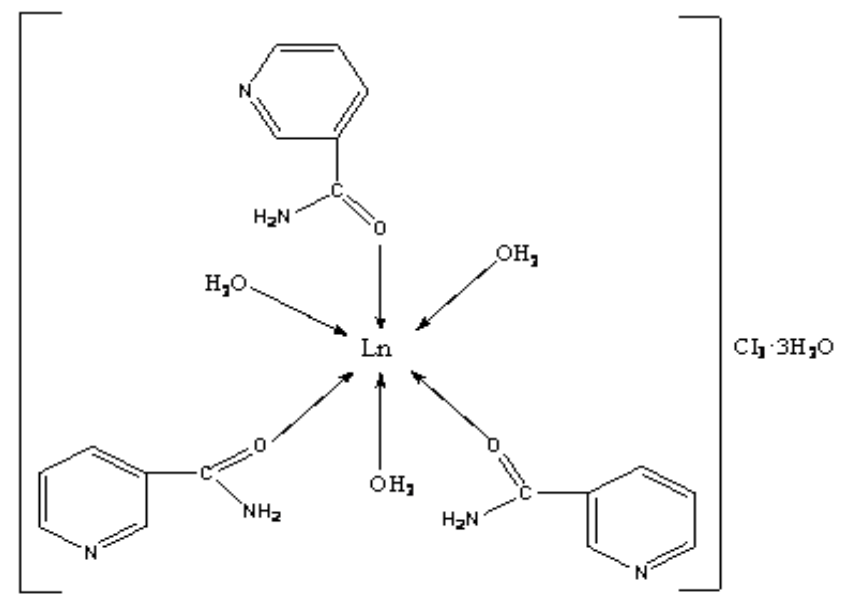

Pис. 5. Схема строения соединения $\mathrm{EuCl}_{3} \cdot 3 \mathrm{C}_{6} \mathrm{H}_{6} \mathrm{~N}_{2} \mathrm{O} \cdot 6 \mathrm{H}_{2} \mathrm{O}$

Атомы кислорода трех молекул воды дополняют координационное число комплексообразователя $\operatorname{Ln}\left(\right.$ где $\left.\operatorname{Ln}=\mathrm{Eu}^{3+}\right)$ до шести.

\section{Выводы}

1. Изотермическим методом при $25^{0} \mathrm{C}$ изучено взаимодействие хлорида европия с никотинамидом и выявлено образование комплексного соединения $\mathrm{EuCl}_{3} \cdot 3 \mathrm{C}_{6} \mathrm{H}_{6} \mathrm{~N}_{2} \mathrm{O} \cdot 6 \mathrm{H}_{2} \mathrm{O}$.

2. Индивидуальность полученного соединения доказана методом ИКспектроскопии и дифференциально-термическим, рентгенографическим анализами.

3. На основании анализа колебательных спектров никотинамида и исследуемого соединения показано, что никотинамид координирован к иону европия через атом кислорода карбонильной группы и предложена структура комплекса.

$$
* * *
$$

1. Киселев Ю. М. Химия координационных соединений / Ю. М. Киселев., Н. А. Добрынина - М.: Академия, - 2007. - С. 320-323.

2. Кинду М.Ф. Синтез и исследование координационных соединений РЗЭ (III) с некоторыми азотсодержащими лигандами и салициловой кислотой: автореф. дис. ... канд. хим наук: 02.00.01 / М. Ф. Кинду. - Москва, 2012. - 21 с.

3. Иванов, В.М. Применение этилендиаминтетраацетата натрия в химическом анализе / В. М. Иванов, Т.Ф. Рудометкина; Моск. гос. ун-т им. М. В. Ломоносова. - Москва, 2019. - С. 19-21.

4. Величко, Н.А. Пищевая химия: учебное пособие / Н. А. Величко, Е.В. Шанина; Краснояр. гос. аграр. ун-т. - Красноярск, 2010. - С. 16 -17.

5. Осмонова С.С. Гетерогенные равновесия в тройной системе хлорид иттрия-никотинамид-вода при 250 С и физико-химические свойства твердых фаз /С.С. Осмонова., Т.Б. Байдинов., Н.О.Орозбаева //Актуальные научные исследования в научном мире. -Вып.12 (32). -Переяслав-Хмельницкий, 2017. -Ч.8. -C.126-135. 